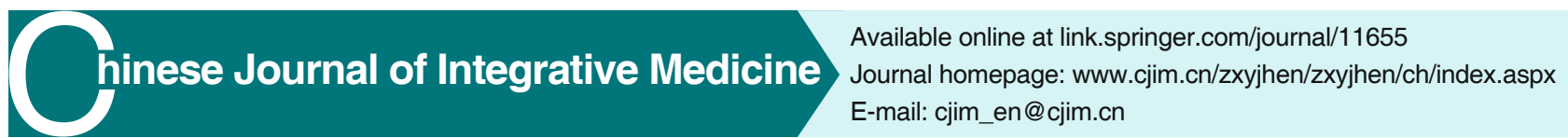

Original Article

\title{
Exploring an Integrative Therapy for Treating COVID-19: A Randomized Controlled Trial*
}

\author{
WANG Jia-bo ${ }^{1}$, WANG Zhong-xia ${ }^{2}$, JING Jing ${ }^{2}$, ZHAO Peng ${ }^{3,4}$, DONG Jing-hui ${ }^{5}$, ZHOU Yong-feng ${ }^{1}$, \\ YANG Guang ${ }^{3}$, NIU Ming ${ }^{1}$, ZHAO Xu' ${ }^{1}$, JIANG Tian-jun ${ }^{3}$, BI Jing-feng ${ }^{6}$, XU Zhe $^{3}$, ZHANG Ping ${ }^{1}$, WU Dan $^{3}$, \\ BAI Zhao-fang', GUO Yu-ming ${ }^{1}$, YU Si-miao², SUN Yong-qiang ${ }^{2}$, ZHANG Zi-teng', ZHAN Xiao-yan", \\ LI Peng-yan ${ }^{1}$, DING Jin-biao ${ }^{6}$, ZHAO Peng-fei ${ }^{8}$, SONG Xue-ai ${ }^{2}$, TANG Jian-yuan ${ }^{7}$, HE Dong-chu', \\ CHEN Zhu ${ }^{4}$, QIN En-qiang ${ }^{3,4}$, WANG Rui-lin ${ }^{2}$, and XIAO Xiao-he ${ }^{1,2}$
}

\begin{abstract}
Objectives: To develop a new Chinese medicine (CM)-based drug and to evaluate its safety and effect for suppressing acute respiratory distress syndrome (ARDS) in COVID-19 patients. Methods: A putative ARDS-suppressing drug Keguan-1 was first developed and then evaluated by a randomized, controlled two-arm trial. The two arms of the trial consist of a control therapy (alpha interferon inhalation, $50 \mu \mathrm{g}$ twice daily; and lopinavir/ritonavir, 400 and $100 \mathrm{mg}$ twice daily, respectively) and a testing therapy (control therapy plus Keguan-1 $19.4 \mathrm{~g}$ twice daily) by random number table at 1:1 ratio with 24 cases each group. After 2-week treatment, adverse events, time to fever resolution, ARDS development, and lung injury on newly diagnosed COVID-19 patients were assessed. Results: An analysis of the data from the first 30 participants showed that the control arm and the testing arm did not exhibit any significant differences in terms of adverse events. Based on this result, the study was expanded to include a total of 48 participants ( 24 cases each arm). The results show that compared with the control arm, the testing arm exhibited a significant improvement in time to fever resolution $(P=0.035)$, and a significant reduction in the development of ARDS $(P=0.048)$. Conclusions: Keguan-1-based integrative therapy was safe and superior to the standard therapy in suppressing the development of ARDS in COVID-19 patients. (Trial registration
\end{abstract} No. NCT 04251871 at www.clinicaltrials.gov)

KEYWORDS COVID-19, SARS-CoV-2, acute respiratory distress syndrome, Chinese medicine

In December 2019, a number of patients with a new type of pneumonia of unknown etiology were detected in Wuhan, China. ${ }^{(1)}$ It was then soon determined that it was a new severe acute respiratory syndrome (SARS) that was caused by a new coronavirus, the SARS-CoV-2 virus. ${ }^{(2)}$ The new pneumonia was later named the Novel 2019 Coronavirus or COVID-19. ${ }^{(3)}$ In 2002-2003, another SARS-causing coronavirus, the SARS-CoV virus, caused one of the most deadly epidemics in recent history. The outbreak of SARS-CoV caused more than 8,000 reported cases and 774 deaths, with a case-fatality rate (CFR) of $7 \%$ in China. ${ }^{(4)}$ Less than a decade later in 2012, another coronavirus, the MiddleEast respiratory syndrome (MERS) virus, the MERSCoV virus, emerged. ${ }^{(5)}$ An outbreak of this virus in 2014 resulted in 662 reported cases and a CFR of $32.97 \%{ }^{(6)}$ Together, these had informed us that coronaviruses represent a new kind of viral pathogens that are characterized by their ability to cause
CThe Chinese Journal of Integrated Traditional and Western Medicine Press and Springer-Verlag GmbH Germany, part of Springer Nature 2020

*Supported by the National Administration of Traditional Chinese Medicine Project (No. 2020ZYLCYJ05-1), the National Natural Science Foundation of China (Nos. 81630100, 81721002, 81930110), China PLA Biosecurity Project (No. 19SWAQ13), China PLA Emergency Project (BWS20J006) and the Beijing Administration of Traditional Chinese Medicine Project (Nos. YJ2020-03, SYFY202011) 1. China Military Institute of Chinese Medicine, The Fifth Medical Center of Chinese PLA General Hospital, Beijing (100039), China; 2. Integrative Medical Center, The Fifth Medical Center of Chinese PLA General Hospital, Beijing (100039), China; 3. Treatment and Research Center for Infectious Diseases, The Fifth Medical Center of Chinese PLA General Hospital, Beijing (100039), China; 4. National Clinical Research Center for Infectious Diseases, Beijing (100039), China; 5. Department of Radiology, The Fifth Medical Center of Chinese PLA General Hospital, Beijing (100039), China; 6. Research Center for Clinical and Translational Medicine, The Fifth Medical Center of Chinese PLA General Hospital, Beijing (100039), China; 7. Sichuan Evidence-Based Medicine Center of Traditional Chinese Medicine Affliated Hospital, Chengdu University of Traditional Chinese Medicine, Chengdu (610075), China; 8. College of Life Science, Beijing University of Chinese Medicine, Beijing (100029), China; 9. Department of Integrated Treatment, General Hospital of Central Theater Command, Wuhan (430070), China

Correspondence to: Prof. XIAO Xiao-he, E-mail: pharmacy302xxh@126.com; Prof. WANG Rui-lin, E-mail: wrl7905@ 163.com; Prof. QIN En-qiang, E-mail: qeq2004@ sina.com DOI: https://doi.org/10.1007/s11655-020-3426-7 
respiratory illnesses that can lead to acute respiratory distress syndrome (ARDS). Since ARDS is known to be associated with a very high CFR, ${ }^{(7,8)}$ they had also revealed the propensity of these pathogens in causing epidemics or pandemics with high CFRs.

The emergence of an epidemic or a pandemic that is caused by a new pathogen poses a unique challenge: the rapid accumulation of a large number of patients without any effective treatments. In the cases of a coronavirus epidemic or pandemic that is associated with a high CFR, the challenge could become an unstoppable and rapid accumulation of casualty. In that regard, the rapid development of an effective treatment to reduce the CFR become the utmost important task. Yet, conventional paradigms for developing effective treatments of such a disease, i.e. the development of new antiviral drugs or vaccines, would take months or even years. ${ }^{(9,10)}$ This realization thus prompted us to consider alternative approaches for the rapid development of an effective treatment for COVID-19. Recently, repurposing or off-label uses of pre-existing non-specific antiviral or host modulating drugs have been proposed as a potential effective strategy for the rapid development of anti-viral therapy. ${ }^{(11,12)}$ Such an approach, however, is still facing the issue of a great uncertainty in term of the chance of success. Meanwhile, a collection of Chinese medicine (CM) drugs had been believed to be effective for treating various types of respiratory distress illnesses (RDI) for thousands of years in China and continued to be an integral part of the modern health care system in China. ${ }^{(13-15)}$ This unique situation prompted us to explore the feasibility of using CM drugs as an effective therapy for COVID-19 and/or for other future coronavirusinduced RDI. We report here the development of a novel $\mathrm{CM}$ drug and the results of a randomized, controlled trial (RCT) that was designed to assess the safety and effect of a therapy that involves the use of this $\mathrm{CM}$ drug.

\section{METHODS}

New CM Drug Development for Suppressing ARDS Development in COVID-19 Patients

Since the high CFRs for these coronaviruscausing epidemics are due to the high incident of ARDS, our mission was to develop a new drug that would hopefully be able to reduce the incident of ARDS in COVID-19 patients and/or patients with other RDI. For both SARS and MERS, there exists a great variability with respect to the responses to the viral infections as well as the final outcomes, ${ }^{(4,7,8)}$ signifying the critical role of the host factors in the responses and the prognoses to such virus infections. Therefore, in order to address this unique challenge, we decided to consider taking an alternative strategy that would target the hosts, rather than the pathogens. Furthermore, considering the presumed safety attribute of some CM compounds, ${ }^{(16)}$ we decided to develop a drug that includes as many of the components as possible so that we could enhance the chance of success in suppressing the development of ARDS, providing that the combination is in line with the fundamental principles of CM.

The new formula was derived from 3 different formulae, Yinqiao Powder (银翅散), Sangju Drink (桑菊饮), and Sanren Decoction (三仁汤), named "Keguan-1" (meaning anti-coronavirus 1 in Chinese) with 7 components: Lonicera japonica Thunb. (Jinyinhua, lot. 19040301) 30 g, Forsythia suspensa (Thunb.) Vahl, (Lianqiao, lot. 19040221) $30 \mathrm{~g}$, Morus alba L. (Sangye, lot. 19045321) $15 \mathrm{~g}$, Chrysanthemum morifolium Ramat. (Juhua, lot. 19040811) $10 \mathrm{~g}$, Coix lacryma-jobi L. var. mayuen (Roman.) Stapf, Yiyiren, lot. 19025161) 30 g, Fritillaria thunbergii Miq. (Zhebeimu, lot. 19041161) $15 \mathrm{~g}$, and Prunus armeniaca L. var. ansu Maxim. (Kuxingren, lot. 19045591) $9 \mathrm{~g}$. The powder versions of the drugs for the 7 components of Keguan-1 were obtained from Beijing Tcmages Pharmaceutical Co. Ltd. (Beijing, China) and mixed in the defined ratio.

Quality control assessments were based on the analyses of the relative amounts of the standard compounds by high-performance liquid chromatography tandem mass spectrometry (HPLC-MS), more details are shown in Appendix 1. A random sample of Keguan-1 drug was analyzed both at the beginning and at the end of the study. The results showed that the quality of Keguan-1 was maintained throughout the study (Figure 1).

\section{Study Design}

A two-arm, randomized, controlled phase I / II trial was conducted to assess the safety and the effect of Keguan-1 in suppressing ARDS dvelopment in COVID-19 patients. The two arms consist of a control therapy that is based on the standard of cares recommended by the China National Health Commission $(\mathrm{CNHC}),{ }^{(17)}$ and a testing therapy in which the patients 

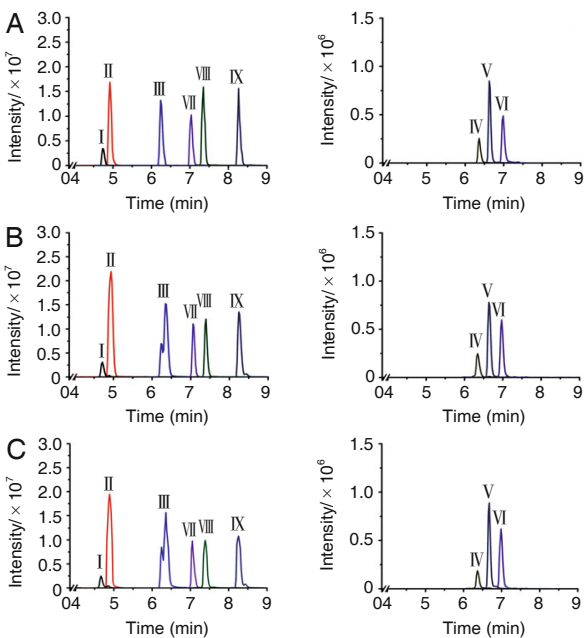

Figure 1. Chromatographs of Mixture of Standard Compounds of Keguan-1

Notes: High-performance liquid chromatography tandem mass spectrometry (HPLC-MS) was used to measure the contents of the 9 control standard compounds for the Chinese medicine components (except for Coix lacryma-jobi L.) in the mixture of control standards for Keguan-1 components (A), in Keguan-1 right after it was made (B) and at the end of the study (C). The control standard compounds for individual components are: Jinyinhua-chlorogenic acid ( I ) and galuteolin (V); Kuxingrenamygdalin ( II); Lianqiao-forsythoside A (III) and forsythin (IX); Sangyerutin (IV); Juhua-chlorogenic acid (I, galuteolin (V), and 3,5-dicaffeoyl quinic acid (VI); Zhebeimu-peimine (VII) and peiminine (VIII). The standard compound (glyceryl trioleate) in Coix lacryma-jobi L. was measured by using a HPLC tandem evaporative light scattering detector. The data were presented in 2 separate graphs with the more abundant ones on the left and the less abundant ones on the right, respectively. Note the difference scales on the $\mathrm{Y}$-axes between the left and the right graphs in each pair of graphs; and that two compounds, chlorogenic acid and galuteolin, are the control standard compounds for more than one component.

were given the new $\mathrm{CM}$ drug in addition to the control therapy. A phase I analysis based on data from the first 30 participants would be performed; if the therapy was proven safe, the trial would then be expanded into a phase II trial to assess the efficacy. The treatment period for each group was 14 days and all the participants were followed-up for 28 days. The trial was approved by the Ethics Board of the Fifth Medical Center of Chinese PLA General Hospital (No. 2020001D), and registered at ClinicalTrials.gov (No. NCT 04251871). The study flowchart is depicted in Figure 2.

\section{Participants Enrollment}

Participants were recruited from "suspected COVID-19 patients" in the Fifth Medical Center of Chinese PLA General Hospital who were newly tested positive for SARS-CoV-2. The "suspected COVID-19 patients" were defined as individuals who had contact history with SARS-CoV-2 positive patient(s) and exhibited at least one of the early symptoms of COVID-19, i.e. fever, cough, aspiration, or abnormal findings in chest $\mathrm{X}$-ray radiography or computer

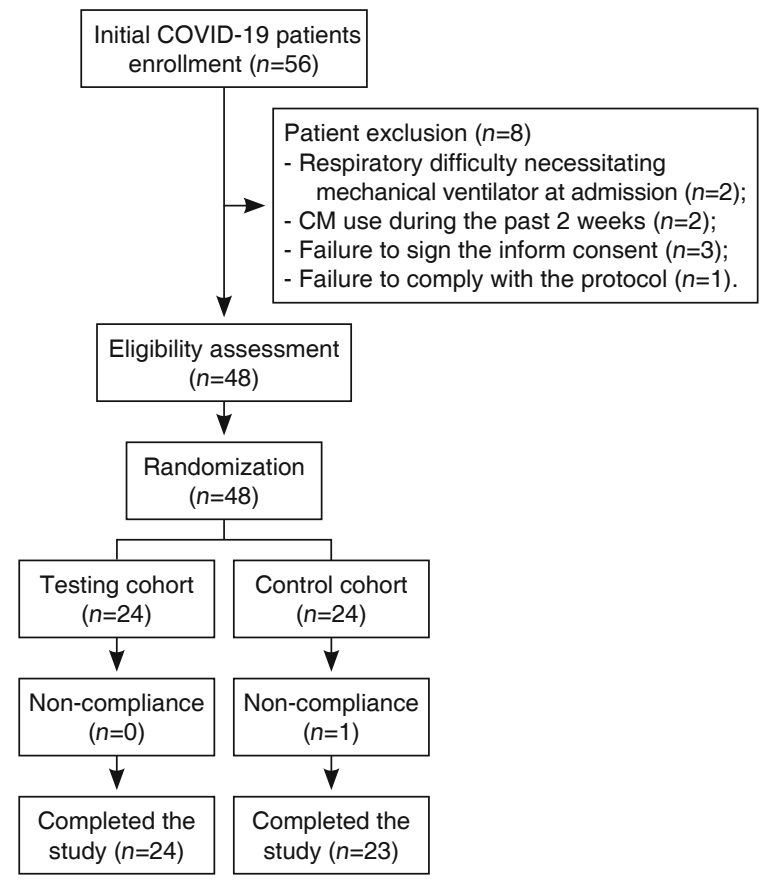

Figure 2. Study Flowchart of Integrative Therapy for COVID-19

tomography (CT) analyses. ${ }^{(17)} \mathrm{A}$ reverse-transcriptasepolymerase-chain-reaction (RT-PCR) assay for SARSCoV-2 RNA was used to identify SARS-CoV-2 positive individuals. The assay kits were provided by the Shanghai BioGerm Medical Biotechnology Co., Ltd. Specifically, for each subject, a sputum swab and a throat swab were tested by two independent entities: the Fengtai District Center for Disease Control and the Beijing Center for Disease Control. An individual was considered SARS-CoV-2 positive if both of his/her samples were tested positive by both testing entities.

Individuals with the following specific conditions were exclued: (1) pregnant or lactating women; (2) respiratory failure necessitating mechanical ventilation; (3) liver failure (total bilirubin $\geqslant 10 \mathrm{mg} / \mathrm{dL}$ and/or severe coagulation disorders); (4) renal function failure [urine $\leqslant 0.5 \mathrm{~m} \cdot \mathrm{kg}^{-1} \cdot \mathrm{h}^{-1}$, creatinine $(\mathrm{Cr})$ or blood urea nitrogen $(B U N) \geqslant 1.5 \times$ upper limits of normal (ULN) although there is adequate circulating blood and cardiac output]; (5) intake of CM or other health supplements during the past 2 weeks; (6) refusal to sign an informed consent form prior to study participation; and (7) unwillingness or inability to comply with the protocol request.

Patient recruitment was initiated on January 22, 2020 , right after the completion of the randomization. 
Patient enrollment was terminated on February 25, 2020, when it was determined that the COVID-19 epidemic in China had subsided.

\section{Randomization and Masking}

Randomization of the cohort assignment for the participants was achieved in the following steps: first, with block $=6$ and ratio of $1: 1$, the random number table (with a total of 150 cases) was generated with SAS (version 9.4). Proc plan statement based on a given seed, by a statistician (who was not involved in data collection or analysis). This led to the arbitrary assignment of the keys into two groups. Following that, patient enrolment was initiated and each eligible participant was given one of these numbers based on the order of being admitted into the participant pool, i.e. the first patient admitted was assigned as 1 , the second as 2 , and so on. This number was then used to determine the cohort affiliation for each participant. The cohort affiliation and treatment assignment for individual participants were masked. However, due to the limited time in preparing the trial, we could not find proper placebo for the control arm. Thus, no placebo was used in the trial.

\section{Clinical Treatments}

Laboratory tests were performed at admission, including a complete blood count and serum biochemistry. All patients were then given the control therapy for 2 weeks. The control therapy was used according to the Directive for the Diagnosis and Recommended Treatments for COVID-19 (version 3$)^{(17)}$ issued by China National Health Commission on January 22, 2020, including the same antiviral treatment (alpha interferon inhalation, $50 \mu \mathrm{g}$ twice daily; and lopinavir/ritonavir, $400 \mathrm{mg}$ and $100 \mathrm{mg}$ twice daily, respectively) and the other supportive treatments. The chief attending physician decided whether other treatments or interventions were given to individual patients, regardless of their cohort affiliations. For the testing arm, each patient was given the same treatments as in the control therapy plus Keguan-1 $19.4 \mathrm{~g}$ twice daily. After 2-week treatment, patients were discharged once they were tested negative for the COVID-19 virus. Discharge conditions included: (1) fever resolution persistent for 3 days; (2) recovery of respiratory tract symptoms; (3) recovery of $\mathrm{X}$-ray radiography; and (4) twice negative RT-PCR results taken $24 \mathrm{~h}$ apart. Patients remained hospitalized were monitored by the same physicians and might be given additional non-antiviral palliative treatments. For those patients who had not been discharged over treatment of
14 days, palliative treatments were given as needed until discharge. All patients were monitored for 28 days (those who were discharged within 28 days were subjected to follow-up monitoring until day 28 after their admission to the study).

\section{Patient Monitoring and Safety Assessment}

During hospitalization, the patients were monitored for a number of parameters by a team of designated nurses who were not aware of the cohort affiliation for the patients for 28 days, starting from the day of admission. Specifically, adverse events, axillary temperature, respiratory distress, and lung injury were monitored. Adverse events were recorded daily in both the treatment period and the follow-up period. The terminologies and grading of severity referred to the Common Terminology Criteria for Adverse Events (CTCAE, version 5.0). ${ }^{(18)}$ Axillary temperatures were measured at 8 a.m. to 9 a.m.; 12 a.m. to 1 p.m.; 4 p.m. to 5 p.m.; and, 8 p.m. to 9 p.m. on a daily basis.

\section{Outcome Assessment}

With the deepening of our understanding of this disease, we realized that the occurrence of ARDS is the most important indicator affecting the fatality rate of COVID-19. Thus, we changed the statistical analysis plan from the original registration as follows: the primary outcome is the incidents of ARDS development; and the secondary outcomes include the time to fever resolution and the recovery of lung injury. These revisions have been updated at ClinicalTrials.gov on May 4, 2020.

Respiratory stress was assessed by monitoring the arterial oxygen tension, inspiratory oxygen fraction, blood oxygen saturation level, continuous positive airway pressure, and positive end-expiratory pressure. ARDS was diagnosed based on Berlin criteria. ${ }^{(19,20)} \mathrm{X}$-ray radiography was used to assess lung injury of the initial 30 patients (phase I ). CT was used for the rest of the study. Radiography or CT was conducted for all patients at admission, day 7 and 14 after the admission. The data were evaluated by a specialist in a blinded fashion.

The radiological recovery of lung injury on X-ray radiographs was defined as the remission of lung injury to the extent of ill-defined hazy increased density in single/both lungs. The radiological recovery of lung injury on chest CT scans was defined as the remission 
of lung injury to the extent of $<5 \%$ involvement of imaging abnormalities, including ground glass opacity, crazy-paving pattern, and consolidation, in each lung lobe. On X-ray radiographs, any imaging findings of ground glass opacities and focal consolidation in each lung were considered non-recovery; on chest $\mathrm{CT}$, any imaging findings of $\geq 5 \%$ involvement in each lung lobe were considered non-recovery.

\section{Statistical Analyses}

The baseline comparison and efficacy evaluation were performed on the per-protocol set. Adverse effects occurred in both the treatment period and the follow-up period were combined to evaluate. Safety was assessed on the safety set. Continuous variables were presented as mean \pm standard deviation $(\bar{x} \pm s)$ or median (range) when the data conforms to normality or non-normality. Comparison between groups was performed using the $t$ test (variance is even) or adjusted t-test (variance is uneven) with normality data, and Wilcoxon test with non-normality data. For those categorical variables, the frequencies or proportions of patients in each category were calculated, and $\chi^{2}$ test was used for comparison between groups. All data were tested on both sides, a $P$-value less than 0.05 was considered statistically significant. Statistics were conducted by a statistician who was blinded to the study grouping. Analyses were done using SAS software, version 9.4.

\section{RESULTS}

\section{Baseline Clinical Characteristics of Study Cohorts}

All but one of the 48 participants received the treatments as designed. The sole non-compliant participant belongs to the control cohort and was excluded from the study. Thus, the control cohort and the testing cohort each has 23 and 24 participants, respectively. A retrospective analysis did not reveal any significant differences between the two cohorts $(P>0.05$, Table 1). Also, there are no differences with respect to the use of other drugs and treatments between the two cohorts ( $P>0.05$, Appendices 2-5).

\section{Adverse Events}

Several mild adverse effects including diarrhea, anorexia, nausea, stomach pain and vomiting, were observed in the control cohort. The addition of Keguan-1 treatment in the testing cohort did not cause new types of adverse events nor any significant
Table 1. Baseline Demographic and Clinical Characteristics of COVID-19 Participants

\begin{tabular}{|c|c|c|c|}
\hline Characteristics & $\begin{array}{l}\text { Testing cohort } \\
\text { ( } 24 \text { cases) }\end{array}$ & $\begin{array}{l}\text { Control cohort } \\
\text { (23 cases) }\end{array}$ & $P$ value \\
\hline Male [Case (\%)] & 14 (58.3) & $12(52.2)$ & 0.772 \\
\hline Mean age (Year) & $46.8 \pm 14.4$ & $51.4 \pm 17.6$ & 0.332 \\
\hline Body mass index $\left(\mathrm{kg} / \mathrm{m}^{2}\right)$ & $24.8 \pm 3.2$ & $25.4 \pm 3.0$ & 0.528 \\
\hline Base line temperature $\left({ }^{\circ} \mathrm{C}\right)$ & $38.0(36.2-39.0)$ & $37.9(36.0-39.1)$ & 0.853 \\
\hline $\begin{array}{l}\text { Onset time of symptom to } \\
\text { enrollment (d) }\end{array}$ & $6.5(3.0-17.0)$ & $8.0(3.0-22.0)$ & 0.779 \\
\hline \multicolumn{4}{|l|}{ Baseline symptoms [Case (\%)] } \\
\hline Fever & $17(70.8)$ & $15(65.2)$ & 0.760 \\
\hline Cough & $15(62.5)$ & $13(56.5)$ & 0770 \\
\hline Fatigue & $7(29.2)$ & $7(30.4)$ & 1.000 \\
\hline $\begin{array}{l}\text { Pre-existing chronic } \\
\text { diseases [Case (\%)] }\end{array}$ & $10(41.7)$ & $8(34.8)$ & 0766 \\
\hline \multicolumn{4}{|l|}{ Laboratory indices } \\
\hline $\begin{array}{l}\text { White blood cell count } \\
\quad\left(\times 10^{9} / \mathrm{L}\right)\end{array}$ & $4.2(2.7-6.8)$ & $5.2(2.8-7.5)$ & 0.105 \\
\hline Neutrophil count $\left(\times 10^{9} / \mathrm{L}\right)$ & $2.7 \pm 0.9$ & $3.1 \pm 1.2$ & 0.299 \\
\hline Platelet count $\left(\times 10^{9} / \mathrm{L}\right)$ & $182.8 \pm 54.7$ & $186.7 \pm 64.6$ & 0.826 \\
\hline CD $4^{+}$count $(\mu L)$ & $400.0 \pm 214.1$ & $462.6 \pm 262.5$ & 0.430 \\
\hline ALT (U/L) & $26.0(13.0-173.0)$ & $20.0(9.0-107.0)$ & 0.096 \\
\hline AST (U/L) & $28.5(14.0-112.0)$ & $27.0(16.0-81.0)$ & 0.447 \\
\hline $\begin{array}{l}\text { Blood urea nitrogen } \\
(\mathrm{mmol} / \mathrm{L})\end{array}$ & $3.7(1.9-7.0)$ & $3.8(2.4-8.8)$ & 0.317 \\
\hline Creatinine $(\mu \mathrm{mol} / \mathrm{L})$ & $76.4 \pm 11.2$ & $79.3 \pm 13.0$ & 0.419 \\
\hline Prothrombin time (s) & $11.9(10.2-13.5)$ & $12.3(10.9-18.1)$ & 0.052 \\
\hline D-dimer (mg/L) & $0.3(0.10-1.3)$ & $0.2(0.1-5.1)$ & 0.798 \\
\hline Procalcitonin (ng/mL) & $0.05(0.02-7.40)$ & $0.05(0.03-0.53)$ & 0.692 \\
\hline Interleukin-6 (pg/mL) & $11.5(1.5-51.7)$ & $8.4(1.5-96.1)$ & 0.904 \\
\hline C-reactive protein (mg/L) & $8.4(0.6-56.9)$ & $7.0(0.4-70.9)$ & 0.874 \\
\hline $\begin{array}{l}\text { Abnormality in chest } \\
\text { radiology [Case (\%)] }\end{array}$ & 20 (83.3) & $16(69.6)$ & 0.318 \\
\hline
\end{tabular}

Notes: quantitative data are presented as $\bar{x} \pm s$ or median (range). *The chest imaging was performed by either X-ray radiography or CT scan. ALT: Alanine aminotransferase; AST: Aspartate aminotransferase

changes in the incidents, duration, or severity of the adverse events compared to those observed in the control cohort (Table 2, Appendix 6). The results of routine blood and biochemical tests at the end of the treatment period did not reveal any significant irregularities for either cohorts (Appendix 7).

\section{Clinical Outcomes}

For the primary outcome, no one developed ARDS in the first 3 days after admission. Over the course of the study, 6 of $23(26.1 \%)$ patients of the control arm and 1 of $24(4.17 \%)$ patients of the testing arm developed ARDS, representing a significant difference in the incidents of ARDS development between the two arms $(P=0.048$, Table 3$)$. Furthermore, 
Table 2. Adverse Events in Phase I Participants with COVID-19 [Case (\%)]

\begin{tabular}{|c|c|c|c|c|c|c|c|c|c|c|c|c|c|}
\hline \multirow{2}{*}{ Adverse events } & \multicolumn{6}{|c|}{ Testing cohort (15 cases) } & \multicolumn{6}{|c|}{ Control cohort (14 cases) } & \multirow{2}{*}{$P$ value } \\
\hline & Total & G1 & G2 & G3 & G4 & G5 & Total & G1 & G2 & G3 & G4 & G5 & \\
\hline Diarrhea & $9(60.0)$ & $6(40.0)$ & $3(20.0)$ & l & l & l & $8(57.1)$ & $7(50.0)$ & $1(7.1)$ & I & I & l & 1.000 \\
\hline Anorexia & $4(26.7)$ & $4(26.7)$ & l & / & l & / & $5(35.7)$ & $5(35.7)$ & l & l & I & l & 0.700 \\
\hline Nausea & $2(13.3)$ & $2(13.3)$ & l & l & l & l & $3(21.4)$ & $3(21.4)$ & / & / & / & l & 0.651 \\
\hline Stomach pain & $2(13.3)$ & $2(13.3)$ & I & l & l & I & $3(21.4)$ & $3(21.4)$ & / & l & I & l & 0.651 \\
\hline Allergic reaction & l & I & l & l & I & l & $1(7.1)$ & l & I & l & 1 & l & 0.483 \\
\hline Sepsis & l & l & l & l & l & I & $1(7.1)$ & l & l & l & l & 1 & 0.483 \\
\hline
\end{tabular}

Notes: G1, mild; G2, moderate; G3, severe; G4, life-threatening; G5, death related

3 of the 6 patients who developed ARDS in the control arm were put on ventilators and 1 of them died on day 24 after admission. The 6 patients in the control arm suffered ARDS for $6,8,11,13,18$, and 20 days, respectively (mean 11.7 days, Table 4). In contrast, the single patient in the testing arm who developed ARDS had ARDS for only 4 days.

Overall, the mean value of time for fever resolution for the control cohort and the testing cohort are 3.0 (1.0-9.0) and $1.5(1.0-5.0)$ days, respectively. This represents a significant reduction in the median value of time to fever resolution for the testing arm ( $1.5 \mathrm{~d}, P=0.035)$.

At the time of admission, $76.6 \%$ of the patients exhibited lung injury. But all of them developed lung injury within 10 days after admission. Over the course of the study, 16 of the $23(69.6 \%)$ patients in control arm and 21 of the $24(87.5 \%)$ patients in testing arm were found to achieve full lung injury recovery judging by the data from X-ray radiography or $\mathrm{CT}$, respectively. Notably, there was a higher (not statistically significant) rate of abnormal radiograph in the testing arm than that in the control arm at baseline; the testing arm achieved higher rate of radiographic recovery after the treatment than the control arm. Thus, the testing arm has a higher rate of lung injury recovery than the control arm, albeit that the difference is not statistically significant $(P=0.168$, Table 3). Typical changes in X-ray radiographs or $\mathrm{CT}$
Table 3. Clinical Courses of COVID-19 Participants [Case (\%)]

\begin{tabular}{lccc}
\hline Indices & $\begin{array}{c}\text { Testing cohort } \\
(24 \text { cases })\end{array}$ & $\begin{array}{c}\text { Control cohort } \\
(23 \text { cases })\end{array}$ & $P$-value \\
\hline Time to fever resolution (d) & $1.5(1.0-5.0)$ & $3.0(1.0-9.0)$ & 0.035 \\
$\begin{array}{c}\text { Time to negative result for } \\
\text { virus (d) }\end{array}$ & $8.0(4.0-22.0)$ & $10.5(4.0-23.0)$ & 0.263 \\
$\begin{array}{l}\text { Lung radiographic recovery* } \\
\text { Occurrence of complications }\end{array}$ & $21(87.5)$ & $16(69.6)$ & 0.168 \\
Any & $1(4.2)$ & $6(26.1)$ & 0.048 \\
ARDS & $1(4.2)$ & $6(26.1)$ & 0.048 \\
Secondary infection & $1(6.7)$ & $3(21.4)$ & 0.330 \\
Acute kidney injury & $2(8.3)$ & $3(13.0)$ & 0.666 \\
Acute cardiac injury & 0 & 0 & \\
Septic shock & 0 & $1(4.3)$ & 0.489 \\
$\begin{array}{l}\text { Death } \\
\text { Use of mechanical ventilation }\end{array}$ & $1(4.2)$ & $3(21.4)$ & 0.348 \\
$\begin{array}{l}\text { Day of mechanical ventilation } \\
\text { No. of patients transferred to }\end{array}$ & $4.0^{\triangle}$ & $11.7 \pm 5.0$ & $\mathrm{~N} / \mathrm{A}$ \\
intensive care unit & 0 & $2(8.7)$ & $\mathrm{N} / \mathrm{A}$ \\
\hline
\end{tabular}

Notes: quantitative data are presented as $\bar{x} \pm s$ or median (range). *The chest imaging was performed by either $\mathrm{X}$-ray radiograph or computer tomography CT scan (Appendices 8-9). ${ }^{\triangle}$ Dada from one patient

scans are depicted in Appendices 8 and 9 .

\section{DISCUSSION}

We have reported here the development of Keguan-1, a new CM drug that was specifically designed for suppressing ARDS development in patients of COVID-19 and/or of other RDI, and the results from

Table 4. Data of COVID-19 Patients Who Developed ARDS

\begin{tabular}{|c|c|c|c|c|c|c|c|c|}
\hline No. & Group & Sex & Age & Pre-existing chronic diseases & $\begin{array}{l}\text { Severity of } \\
\text { ARDS }\end{array}$ & Treatment & $\begin{array}{r}\text { Duration of } \\
\text { ARDS (d) }\end{array}$ & Outcome \\
\hline 13 & A & Female & 79 & NA & Severe & Invasive mechanical ventilation & 18 & Death \\
\hline 21 & A & Male & 33 & NA & Mild & High-flow nasal oxygen & 11 & Discharge \\
\hline 23 & $A$ & Male & 77 & Chronic kidney disease and hypertension & Moderate & Non-invasive mechanical ventilation & 13 & Discharge \\
\hline 29 & A & Female & 60 & Asthma & Mild & High-flow nasal oxygen & 6 & Discharge \\
\hline 39 & B & Male & 34 & NA & Moderate & Non-invasive mechanical ventilation & 4 & Discharge \\
\hline 40 & A & Female & 74 & Atherosclerosis & Moderate & High-flow nasal oxygen & 8 & Discharge \\
\hline 41 & $A$ & Male & 78 & Hypertension, diabetes and prostatic cancer & Moderate & Non-invasive mechanical ventilation & 20 & Discharge \\
\hline
\end{tabular}


a randomized, controlled trial aiming at assessing the safety and efficacy of this new drug. The results of the clinical trial showed that Keguan-1, when used in conjunction with the treatments of a control therapy, did not cause any significant alterations with respect to the types, the incidents, or the severities of adverse events, demonstrating that this new CM-based drug is safe when used under the specific clinical setting. In terms of efficacy, the drug appeared to have beneficial effects for both the primary and the secondary endpoints. Specifically, the addition of Keguan-1 to the control therapy led to: a significant reduction in the incidents of ARDS development $(P=0.048)$; a significant shortening in time to fever resolution $(P=0.035)$; and a trend of improvement (albeit not statistically significant) in the incident of lung injury recovery. Notably, the single case of ARDS in the testing arm had a shorter duration on the ventilator and a shorter time to full recovery than any of the 6 ARDS cases in the corresponding control arm, suggesting a potential beneficial effect in the recovery even after the development of ARDS. Together, these results have shown that Keguan-1 could be used safely to suppress ADRS in COVID-19 patients. In addition, the findings have also provided support for the claims that these CM components are safe for human use and could have beneficial effects for patients experiencing respiratory distress.

Keguan-1 was developed specifically for suppressing the development of ARDS by targeting the host. It was based on an anticipation that SARSCoV-2 could lead to a respiratory illness that is similar to those caused by SARS-CoV and MERS-CoV, which are characterized by their propensity of outbreak into epidemics with high CFRs and the critical role of the host factor for prognosis. ${ }^{(4-6)}$ Today, COVID-19 has evolved not only in regional epidemics, but a global pandemic. ${ }^{(21)}$ Although its CFR is lower than those of SARS and MERS, ${ }^{(22,23)}$ but it is still terribly high, particularly considering its highly contagious nature and its potential global implication. ${ }^{(24)}$ Significantly, there indeed exists a great variability in the responses and prognosis among COVID-19 patients, ${ }^{(25-27)}$ confirming the suspicion that the host factors paly a very important role in the clinical course of the disease. The mechanism of Keguan-1 in the treatment of COVID-19 may be involved in modulating immune system. Poon, et $\mathrm{al}^{(28)}$ found that the Sangju Drink had immunomodulating effects in healthy volunteers, including significant increase of the T-lymphocyte CD4/CD8 ratio. Investigations also found that the herbal ingredients in Keguan-1 and their active components have a potent anti-inflammatory effect. For instance, the major component in Forsythia suspensa, forsythiaside, was previously demonstrated to have dosedependent protective effects on lung injury by attenuating infiltration of inflammatory cells and suppressing tumor necrosis factor $\alpha$, interleukin- 6 and - $1 \beta$ production through nuclear factor kappa-B. ${ }^{(29)}$ The major component in Lonicera japonica, neochlorogenic acid, could prevent excessive macrophage-mediated inflammatory responses as an activator upregulating AMP-activated protein kinase/nuclear factor erythroid 2-related factor 2 signal transduction. ${ }^{(30)}$ Together, these new information have validated the merit of our original design. Importantly, COVID-19 is still spreading in many places throughout the globe. Yet, no effective treatments or vaccines are in sight. ${ }^{(24,25,31)}$ In the regards, any effective therapy for COVID-19 would potentially have a life-saving value.

In addition, Keguan-1 might suppress ARDS development by targeting the host. Given that ARDS development is the shared key development that leads to a fatal outcome in many respiratory distress illnesses, ${ }^{(23,26)}$ this drug could provide an effective strategy for suppressing ARDS development in respiratory distress illness of other cause and hence a new paradigm for coping with the challenge posed by respiratory distress illnesses due to the newly emerging pathogens. Additionally, the results of this study have lent support for the claim that the components used to make Keguan-1 have excellent safety attributes. Therefore, it is conceivable that its combined use with certain drugs could also be safe. In particular, it may be used to enhance the effect of other anti-SARS-CoV-2 drugs, which should be developed in the future.

The study, however, does have its limitations. First, the cohort sizes are quite small, due to a failure in recruiting a large number of COVID-19 patients before the end of the outbreak in China. The results in this paper should be interpreted for caution. However, it is conceivable that similar studies but with larger scales can be carried out in other countries where COVID-19 cases are still on the rise. Second, X-ray radiography, rather than computer tomography, was used at the early phase of the study to assess the degrees of lung injury due to a logistic issue in setting up the computer tomography system inside the designated confinement unit in a timely manner. In addition, the study did not include a placebo arm due to an ethical consideration. 


\section{Conflict of interest}

None declared.

\section{Author Contributions}

Wang JB, Wang ZX, and Jing J conducted the study and wrote the manuscript, they contributed equally to this work as cofirst authors. Wang ZX, Jing J, Zhao P, Dong JH, Zhou YF, Yang G, Jiang TJ, Xu Z, Wu D, Sun YQ, Wang JB, He DC, and Chen Z collected data and provided technical assistance. Jing J, Yu SM, Niu M, Zhao X, Zhang P, Song XA, Bi JF, Bai ZF, Guo YM, Zhao X, Zhao PF, Tang JY, and Li PY analyzed data. Wang JB, Wang RL, Qin $\mathrm{EQ}$, and Xiao $\mathrm{XH}$ initiated and oversaw the study.

\section{Acknowledgements}

The authors acknowledge Academician WANG Fu-sheng for his advice to the trial.

\section{Data Sharing Statements: See Appendix 10.}

Electronic Supplementary Material: Supplementary material is available in the online version of this article at https://doi. org/10.1007/s11655-020-3426-7.

\section{REFERENCES}

1. Wuhan Municipal Health Commission. Report of clustering pneumonia of unknown etiology in Wuhan City. Dec 31, 2019. Availabe at: http://wjw.wuhan.gov.cn/front/web/ showDetail/2019123108989 Accessed Feb 29, 2020).

2. China National Emergency Office. Prevention and control of the pneumonia caused by a new coronavirus. Jan 21, 2020. Availabe at: http://www.nhc.gov.cn/yjb/s7860/202001/d9570ł3a52614113ae0093 df51509684.shtml (Accessed Feb 29, 2020).

3. WHO. Naming the coronavirus disease (COVID-19) and the virus that causes it. Availabe at: https://www.who.int/emergencies/diseases/novelcoronavirus-2019/technical-guidance/naming-the-coronavirus-disease(covid-2019)-and-the-virus-that-causes-it (Accessed Feb 29, 2020).

4. Anderson RM, Fraser C, Ghani AC, Donnelly CA, Riley S, Ferguson $\mathrm{NM}$, et al. Epidemiology, transmission dynamics and control of SARS: the 2002-2003 epidemic. Philos Trans R Soc Lond B Biol Sci 2004;359:1091-1105.

5. Mackay IM, Arden KE. MERS coronavirus: diagnostics, epidemiology and transmission. Virol J 2015;22:222.

6. Al-Omari A, Rabaan AA, Salih S, Al-Tawfiq JA, Memish ZA. MERS coronavirus outbreak: implications for emerging viral infections. Diagn Microbiol Infect Dis 2019;93:265-285.

7. Ware LB, Matthay MA. The acute respiratory distress syndrome. N Engl J Med 2000;342:1334-1349.

8. Bauer TT, Ewig S, Rodloff AC, Müller EE. Acute respiratory distress syndrome and pneumonia: a comprehensive review of clinical data. Clin Infect Dis 2006;43:748-756.

9. Labonté $P$, Seidah NG. Emerging viruses: risk of pandemic. Expert Rev Anti Infect Ther 2008;6: 581-583.

10. Adalja A, Inglesby T. Broad-spectrum antiviral agents: a crucial pandemic tool. Expert Rev Anti Infect Ther 2019;17:467-470.

11. Kil HR, Lee JH, Lee KY, Rhim JW, Youn YS, Kang JH. Early corticosteroid treatment for severe pneumonia caused by 2009 H1N1 influenza virus. Crit Care 2011;15:413.

12. Lee KY. Pneumonia, acute respiratory distress syndrome, and early immune-modulator therapy. Int J Mol Sci 2017;18:388.

13. Chan K. Progress in traditional Chinese medicine. Trends Pharmacol Sci 1995;16:182-187.

14. Chen YB, Liu $Q$, Xie H, Yin SM, Wu L, Yu XH, et al. Is Chinese medicine injection applicable for treating acute lung injury and acute respiratory distress syndrome? A systematic review and metaanalysis of randomized controlled trials. Chin J Integr Med 2019. [Epub ahead of print]

15. Luo H, Tang QL, Shang YX, Liang SB, Yang M, Robinson N, et al. Can Chinese medicine be used for prevention of corona virus disease 2019 (COVID-19)? A review of historical classics, research evidence and current prevention programs. Chin J Integr Med 2020;26:243-250.

16. Li N, Gao F. Viewpoint regarding "concise" of Jing-Fang from case. China J Chin Mater Med (Chin) 2019;44:3899-3902.

17. National Health Commission of the People's Republic of China Directive for the diagnosis and recommended treatments for COVID-19 (3rd ed). Jan 22, 2020. Available at: http://www.nhc.gov. cn/yzygj/s7653p/202001/f492c9153ea9437bb587ce2ffcbee1fa. shtml (Accessed Jan 23, 2020).

18. U.S. Department of Health and Human Services. Common terminology criteria for adverse events (CTCAE) Version 5.0. Available at: https://ctep.cancer.gov/protocoldevelopment/electronic_ applications/docs/CTCAE_v5_Quick_Reference_5x7.pdf.

19. ARDS Definition Task Force, Ranieri VM, Rubenfeld GD, Thompson BT, Furguson ND, Caldwell E, et al. Acute respiratory distress syndrome: the Berlin Definition. JAMA 2012;307:2526-2533.

20. Riviello ED, Kiviri W, Twagirumugabe T, Mueller A, BannerGoodspeed VM, Officer L, et al. Hospital incidence and outcomes of the acute respiratory distress syndrome using the kigali modification of the Berlin definition. Am J Respir Crit Care Med 2016;193:52-59.

21. Habibzadeh $P$, Stoneman EK. The novel coronavirus: a bird's eye view. Int J Occup Environ Med 2020;11:65-71.

22. Huang $\mathrm{C}$, Wang $\mathrm{Y}$, Li X, Ren L, Zhao J, Hu Y, et al. Clinical features of patients infected with 2019 novel coronavirus in Wuhan, China. Lancet 2020;395:497-506.

23. Yang XB, Yuan $Y, X u J Q$, Shu $H$, Xia J, Liu H, et al. Clinical course and outcomes of critically ill patients with SARS-CoV-2 pneumonia in Wuhan, China: a single-centered, retrospective, observational study. Lancet Respir Med 2020:30079-5. [Epub ahead of print]

24. Li Q, Guan X, Wu P, Wang X, Zhou L, Tong Y, et al. Early transmission dynamics in Wuhan, China, of novel coronavirusinfected pneumonia. N Engl J Med 2020;382:1199-1207.

25. Cao B, Wang Y, Wen D, Liu W, Wang J, Fan G, et al. A trial of lopinavir-ritonavir in adults hospitalized with severe COVID-19. N Engl J Med 2020:NEJMoa2001282. [Epub ahead of print]

26. Peeri NC, Shrestha N, Rahman MS, Zaki R, Tan Z, Bibi S, et al. The SARS, MERS and novel coronavirus (COVID-19) epidemics, the newest and biggest global health threats: what lessons have we learned? Int J Epidemiol 2020:dyaa033. [Epub ahead of print]

27. Guan WJ, Ni ZY, Hu Y, Liang WH, Ou CQ, He JX, et al. Clinical characteristics of coronavirus disease 2019 in China. N Engl J Med 2020:NEJMoa2002032. [Epub ahead of print]

28. Poon PMK, Wong CK, Fung KP, Fong CYS, Wong ELY, Lau JTF, et al. Immunomodulatory effects of a traditional Chinese medicine with potential antiviral activity: a self-control study. Am J Chin Med 2006;34:13-21.

29. Cheng L, Li F, Ma R, Hu XP. Forsythiaside inhibits cigarette smokeinduced lung inflammation by activation of Nrf2 and inhibition of NF- $к$ B. Int Immunopharmacol 2015;28:494-499.

30. Park SY, Jin ML, Yi EH, Kim Y, Park G. Neochlorogenic acid inhibits against LPS-activated inflammatory responses through upregulation of Nrf2/HO-1 and involving AMPK pathway. Environ Toxicol Pharmacol 2018;62:1-10.

31. Čivljak R, Markotić A, Kuzman I. The third coronavirus epidemic in the third millennium: what's next? Croat Med J 2020;61:1-4.

(Accepted June 26, 2020; First Online July 16, 2020) Edited by YUAN Lin 